\title{
VERTICAL SHEAR MIXING IN STELLAR RADIATIVE ZONES
}

\author{
V. Prat ${ }^{1,2}$, J. Guilet ${ }^{2}$, M. Vialler ${ }^{2}$ and E. Müller ${ }^{2}$
}

\begin{abstract}
Jean-Paul Zahn's formalism for vertical shear mixing is used in several stellar evolution codes, but the physics of the shear instability in stellar radiative zones is still not completely understood. Over the last few years, numerical simulations have provided new constraints on the shear instability, including the effect of thermal diffusion and chemical stratification. We present here new simulations that show the effect of viscosity on the vertical turbulent transport due to the shear instability.
\end{abstract}

\section{Introduction}

Stellar models are used in various domains of astrophysics because they provide us with a link between observable quantities and fundamental parameters of stars, which can be used to constrain other objets, such as galaxies or planetary systems. However, these models present large uncertainties due to our lack of understanding of stellar evolution. In particular, dynamical processes, such as rotation, magnetism, turbulence and waves, are key processes of stellar evolution for they transport angular momentum and mix chemical elements. Because of their multidimensional character, they cannot be self-consistently resolved in unidimensional stellar evolution calculations and thus need to be prescribed.

Jean-Paul Zahn was a pioneer in the effort to improve the predictive power of stellar evolution by properly modeling these processes. In particular, he proposed in his famous paper Zahn (1992) a phenomenological model for the turbulent transport induced by the shear instability. The main feature of this model is that it takes the effect of thermal diffusion on the stability of shear flows into account. The model has been extended by adding physical ingredients such as gradients of

${ }^{1}$ Laboratoire AIM Paris-Saclay, CEA/DRF - CNRS, Université Paris Diderot, IRFU/SAp Centre de Saclay, 91191 Gif-sur-Yvette, France

2 Max-Planck Institut für Astrophysik, Karl-Schwarzschild-Str. 1, 85748, Garching bei München, Germany 
mean molecular weight or horizontal turbulence (Maeder 1995; Maeder \& Meynet 1996; Maeder 1997; Talon \& Zahn 1997) and is still used in various forms in stellar evolution codes.

The problem of such models is that they only give the order of magnitude of the transport coefficient and they rely on strong assumptions that need to be tested. This has become crucial since various observational constraints suggest that shear mixing as implemented in stellar evolution codes is not efficient enough. These constraints are mainly split into two categories. First, abundance measurements using spectrometry (e.g. Hunter et al.2009) show anomalies that are not accounted for by the current description of shear mixing. In particular, synthetic stellar populations do not predict the existence of nitrogen-enriched slow rotators, although such stars are observed (Brott et al. 2011; Potter et al. 2012). Second, asteroseismic measurements of the rotation profile of giant and subgiant stars (Beck et al. 2012; Mosser et al. 2012; Deheuvels et al. 2012, 2014, 2015) show that the rotation contrast between the core and the surface is much lower than what is predicted by stellar evolution codes (Ceillier et al. 2012; Eggenberger et al. 2012; Marques et al. 2013).

Additional processes, such as magnetic field (e.g. Meynet et al. 2011) and inertial gravity waves (e.g. Fuller et al. 2014), are often invoked as potential sources for the missing transport of angular momentum and chemical elements. However, shear mixing is still not completely understood, and missing ingredients may have a strong impact on the transport. In this context, numerical simulation appears as an essential tool to provide new constraints on turbulent transport processes. One of the limiting factors is that stellar radiative zones have a very high thermal diffusivity, which makes numerical computations without approximation prohibitively expensive. To solve the problem, Lignières (1999) proposed an asymptotic development of the Boussinesq equations in this regime, the smallPéclet number (SPN) approximation, also called low-Péclet-number approximation (LPN), that drastically reduces the computational cost.

Using an imposed uniform shear profile, Prat \& Lignières (2013) validated for the first time the SPN approximation in a non-linear regime and showed that Zahn (1992) is consistent with this approximation. Then, Prat \& Lignières (2014) investigated the effect of a gradient of mean molecular weight on the transport. Using a sinusoidal forcing, Garaud et al. (2015) studied the stability of the flow, and Garaud \& Kulenthirarajah (2016) measured the transport generated by the shear instability in such a flow. Their conclusion is that Zahn (1992) is a good approximation in the moderately stratified regime, whereas in the strongly stratified regime, viscosity may play a role.

In the work presented here, we investigated the effect of viscosity on turbulent transport thanks to new simulations detailed in Prat et al. (2016). The configuration of the considered flow is presented in Section 2. In Section 3 we interpret the results of our simulations on the transport of chemical elements. In Section 4 we show the relation between turbulent viscosity and turbulent diffusivity. Finally, we conclude in Section 5 . 


\section{Flow configuration}

We considered a flow configuration similar to Prat \& Lignières (2013) and Prat \& Lignières (2013), that is with a uniform vertical velocity shear and a uniform vertical temperature gradient (which is equivalent to an entropy gradient in the Boussinesq approximation). The simulations were performed with the Snoopy code (Lesur \& Longaretti 2005), using the shearing-box formalism. To simplify the interpretation, we considered only the chemically neutral case.

In the SPN approximation, the dimensionless governing equations are

$$
\begin{aligned}
\vec{\nabla} \cdot \vec{v} & =0, \\
\frac{\partial \vec{v}}{\partial t}+(\vec{v} \cdot \vec{\nabla}) \vec{v} & =-\vec{\nabla} P+\operatorname{RiPe\psi } \vec{e}_{z}+\frac{1}{R e} \Delta \vec{v}, \\
v_{z} & =\Delta \psi, \\
\frac{\partial \mu}{\partial t}+\vec{v} \cdot \vec{\nabla} \mu & =\frac{1}{P e_{\mu}} \Delta \mu,
\end{aligned}
$$

where $\vec{v}$ is the dimensionless velocity, $P$ the dimensionless pressure, $\psi$ the normalised temperature fluctuations, $\vec{e}_{z}$ the vertical unit vector pointing upwards, and $\mu$ the chemical concentration of a passive scalar. The dimensionless parameters involved in these equations are (i) the Richardson-Péclet number $R i P e=$ $N^{2} L^{2} /(S \kappa)$, where $N$ is the Brunt-Väisälä frequency that accounts for stable stratification, $S=\mathrm{d} V / \mathrm{z}$ the shear rate, $L$ the size of the numerical domain, and $\kappa$ the thermal diffusivity of the fluid; (ii) the Reynolds number $R e=S L^{2} / \nu$, which measures the effect of the viscosity $\nu$; and (iii) the chemical Péclet number $P e_{\mu}=S L^{2} / \kappa_{\mu}$, which measures the effect of the chemical diffusivity $\kappa_{\mu}$.

\section{Effect of viscosity on the transport of chemical elements}

The turbulent diffusion coefficient proposed by Zahn (1992) has no explicit dependence on viscosity, but it assumes that the turbulence is fully developed. This is equivalent to say that the turbulent Reynolds number $R e_{\ell}=S \ell^{2} / \nu$, where $\ell$ is a typical turbulent length scale, is much larger than a critical value $R e_{\mathrm{c}}$. In Zahn's model, $\ell$ is such that $R i P e_{\ell}$, where $P e_{\ell}=S \ell^{2} / \kappa$, is equal to its critical value $(R i P e)_{c}$, which is of the order of one. The Reynolds number can be written

$$
R e_{\ell}=\frac{(\operatorname{RiPe})_{\mathrm{c}}}{\operatorname{RiPr}}
$$

and the condition for a fully developed turbulence becomes

$$
\operatorname{RiPr}<(\operatorname{RiPr})_{\mathrm{c}}
$$

where $\operatorname{Pr}=\nu / \kappa$ is the Prandtl number. This condition has the same form as the energy stability limit predicted by Garaud et al. (2015). 


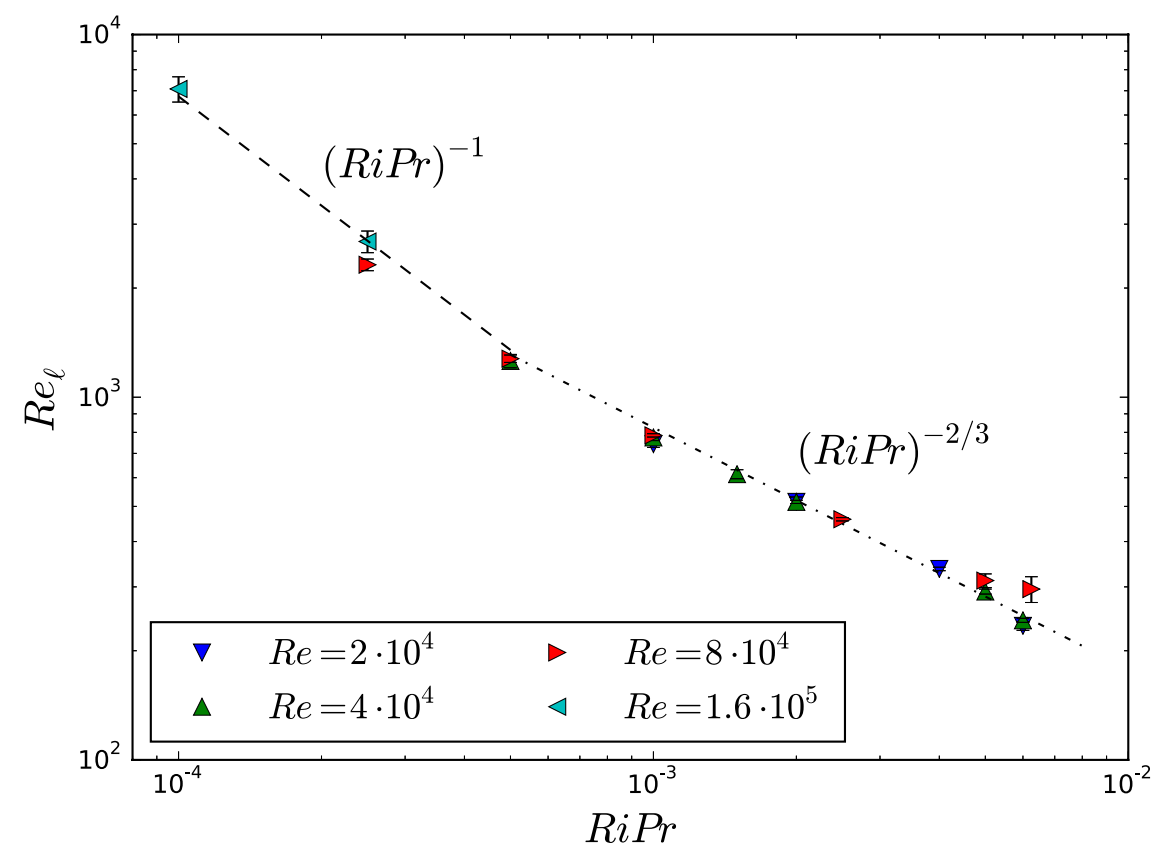

Fig. 1. Turbulent Reynolds number $R e_{\ell}$ as a function of $R i P r$.

A test of the relation (3.1) is shown in Figure 1. First, one can see that for $\operatorname{RiPr}<5 \cdot 10^{-4}$, the expected scaling law is satisfied, which suggests that there is an asymptotic regime at large values of the turbulent Reynolds number $\left(R e_{\ell}>\right.$ $\left.R e_{\mathrm{c}} \sim 10^{3}\right)$, as predicted by Zahn (1992). Second, this scaling law is not satisfied for larger values of $R i P r$. Nevertheless, there is always a one-to-one relation between the turbulent Reynolds number and RiPr. A last important point is that turbulent simulations could not be obtained for values of $\operatorname{RiPr}$ greater than $7 \cdot 10^{-3}$. This qualitatively validates the energy stability limit by Garaud et al. $(2015)$ and suggests that $(\operatorname{RiPr})_{\mathrm{c}} \simeq 7 \cdot 10^{-3}$.

The dependence of the turbulent diffusion coefficient on $\operatorname{RiPr}$ also shows different regimes, as illustrated in Figure 2. For $\operatorname{RiPr}<5 \cdot 10^{-4}$, the quantity $D_{\mathrm{t}} /\left(\kappa R i^{-1}\right)$ seems to tend to a constant value, which means that $D_{\mathrm{t}} \propto \kappa / R i$. This corresponds to the asymptotic regime described by Zahn (1992). Our simulations give an estimate of the proportionality constant $\alpha \simeq 3.34 \cdot 10^{-2}$, which is significantly smaller than originally proposed in the model. When $\operatorname{RiPr}$ becomes close to the critical value, the diffusion coefficient rapidly decreases and finally vanishes at the point where there is no more turbulence. Between these two regimes, $D_{\mathrm{t}} /\left(\kappa R i^{-1}\right)$ has a maximum around $\operatorname{RiPr}=3 \cdot 10^{-3}$. To account for this non-monotonic behaviour, we can use a quadratic fit

$$
D_{\mathrm{t}}=\frac{\kappa}{R i}\left[\alpha+\beta R i P r-\gamma(\operatorname{RiPr})^{2}\right],
$$

where $\beta=18.8$ and $\gamma=2.86 \cdot 10^{3}$. 


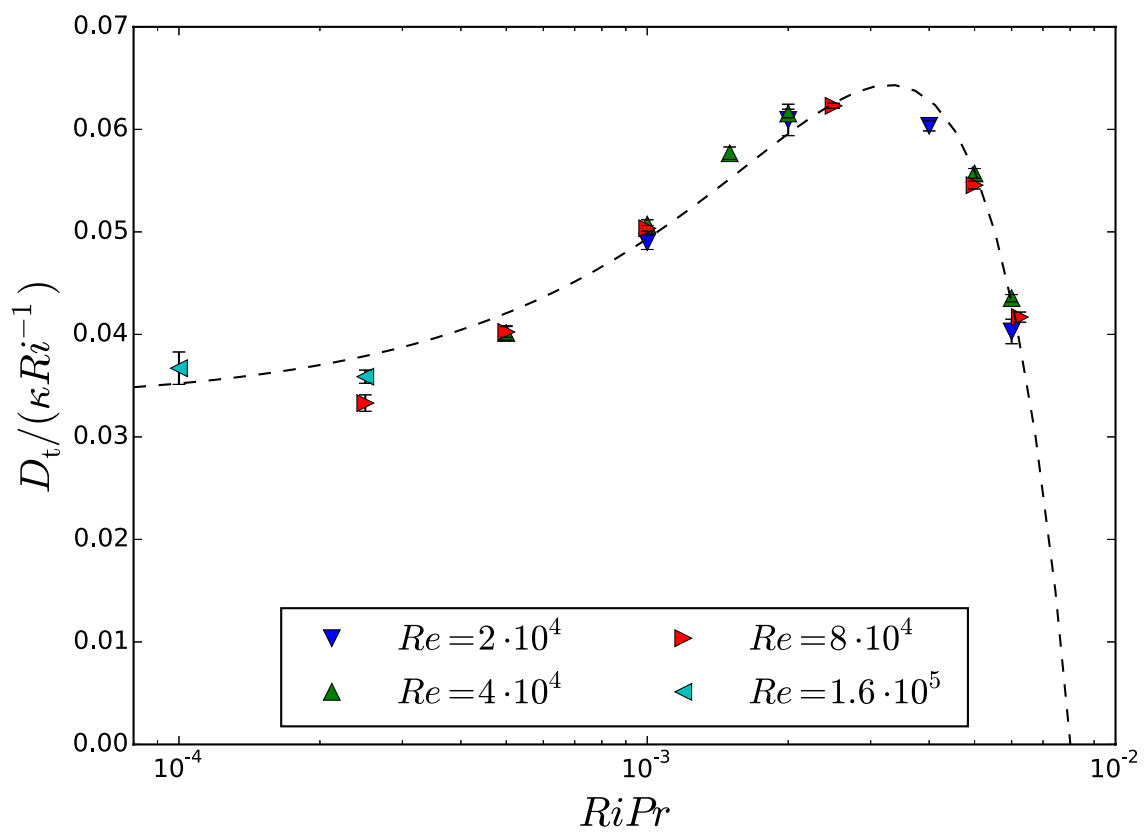

Fig. 2. $D_{\mathrm{t}} /\left(\kappa R i^{-1}\right)$ as a function of $\operatorname{RiPr}$. The dashed line is the quadratic fit given in the text.

\section{Turbulent viscosity and turbulent diffusivity}

In stellar evolution codes, turbulent viscosity is usually considered equal to turbulent diffusivity. It means that the transport of angular momentum is as efficient as the transport of chemical elements. Our simulations allowed us to test this assumption. Figure 3 shows the relation between the two transport coefficients. For simulations with a weak turbulence (that is at $R i P r$ close to its critical value), the ratio between turbulent viscosity and turbulent diffusivity is close to 1 . For more turbulent simulations, the ratio is closer to 0.8. This shows that the assumption that many codes make is not rigourously correct. Though, the impact on stellar evolution models is probably limited, since turbulent viscosity remains of the same order of magnitude as turbulent diffusivity.

\section{Conclusion}

Our new simulations provide us with additional constraints on the efficiency of vertical shear mixing in stellar radiative zones. Concerning the diffusion of chemical elements, our results confirm that the number $R i P r$ is the key parameter. In the regime of low $R i P r$, we found that the model by Zahn (1992) is valid, but with a significantly smaller proportionality constant. In the regime of high $\mathrm{RiPr}$ $\left(>7 \cdot 10^{-3}\right)$, in contrast, viscosity is able to damp turbulent motions, so that the turbulent diffusion coefficient vanishes. This means that viscosity can create dead zones where there is no turbulent transport, which would significantly reduce the 


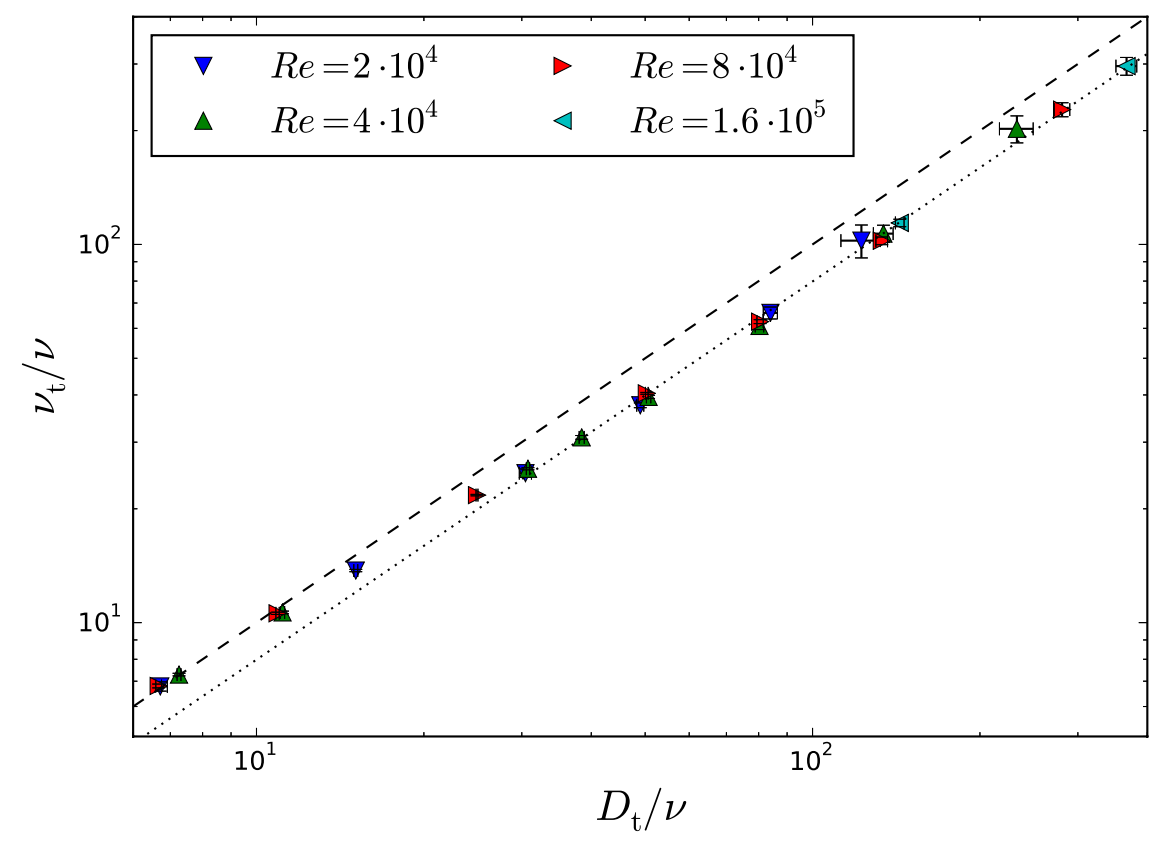

Fig. 3. Turbulent viscosity $\nu_{\mathrm{t}}$ as a function of turbulent diffusivity $D_{\mathrm{t}}$. The dotted line is the curve $\nu_{\mathrm{t}}=0.8 D_{\mathrm{t}}$ and the dashed line is the curve $\nu_{\mathrm{t}}=D_{\mathrm{t}}$.

global transport. We provide a new empirical prescription that is in agreement with our simulations and needs to be implemented in stellar evolution codes, but we can already say that this new prescription does not help to reduce the discrepancy between theory and observations.

We also showed that the transport coefficient for angular momentum is close to the turbulent diffusion coefficient, though they are not rigourously equal. Thus, our conclusion on the lack of efficiency of shear mixing also stands for the transport of angular momentum.

To go further, one may invoke additional transport processes to generate more transport, such as the magneto-rotational instability (MRI, see e.g. Menou \& Le Mer 2006; Guilet \& Müller 2015). However, additional physical ingredients might increase the efficiency of shear mixing. Among them is horizontal turbulence, which can be notably induced by the Coriolis acceleration or by a horizontal shear. According to Talon \& Zahn (1997), it is able to enhance vertical mixing. The presence of a magnetic field could also make stable configurations become unstable (Lecoanet et al. 2010). These potential effects have to be tested with numerical simulations similar to those presented here.

\section{References}

Beck, P.G., Montalban, J., Kallinger, T., et al., 2012, Nature, 481, 55

Brott, I., Evans, C.J., Hunter, I., et al., 2011, A\&A, 530, A116 
Ceillier, T., Eggenberger, P., García, R.A., \& Mathis, S. 2012, Astron. Nach., 333, 971

Deheuvels, S., Ballot, J., Beck, P.G., et al., 2015, A\&A, 580, A96

Deheuvels, S., Doğan, G., Goupil, M.J., et al., 2014, A\&A, 564, A27

Deheuvels, S., García, R.A., Chaplin, W.J., et al., 2012, ApJ, 756, 19

Eggenberger, P., Montalbán, J., \& Miglio, A., 2012, A\&A, 544, L4

Fuller, J., Lecoanet, D., Cantiello, M., \& Brown, B., 2014, ApJ, 796, 17

Garaud, P., Gallet, B., \& Bischoff, T., 2015, Phys. Fluids, 27, 084104

Garaud, P., \& Kulenthirarajah, L., 2016, ApJ, 821, 49

Guilet, J., \& Müller, E., 2015, MNRAS, 450, 2153

Hunter, I., Brott, I., Langer, N., et al., 2009, A\&A, 496, 841

Lecoanet, D., Zweibel, E.G., Townsend, R.H.D., \& Huang, Y.-M., 2010, ApJ, 712, 1116

Lesur, G., \& Longaretti, P.-Y., 2005, A\&A, 444, 25

Lignières, F., 1999, A\&A, 348, 933

Maeder, A., 1995, A\&A, 299, 84

Maeder, A., 1997, A\&A, 321, 134

Maeder, A., \& Meynet, G., 1996, A\&A, 313, 140

Marques, J.P., Goupil, M.J., Lebreton, Y., et al., 2013, A\&A, 549, A74

Menou, K., \& Le Mer, J., 2006, ApJ, 650, 1208

Meynet, G., Eggenberger, P., \& Maeder, A., 2011, A\&A, 525, L11

Mosser, B., Goupil, M.J., Belkacem, K., et al., 2012, A\&A, 548, A10

Potter, A.T., Tout, C.A., \& Brott, I., 2012, MNRAS, 423, 1221

Prat, V., Guilet, J., Viallet, M., \& Müller, E., 2016, A\&A, 592, A59

Prat, V., \& Lignières, F., 2013, A\&A, 551, L3

Prat, V., \& Lignières, F., 2014, A\&A, 566, A110

Talon, S., \& Zahn, J.-P., 1997, A\&A, 317, 749

Zahn, J.-P., 1992, A\&A, 265, 115 